\title{
Retinal and Choroidal Manifestations of Selected Systemic Diseases Eds: Arevalo J F (2013) ISBN: 978-1-4614-3645-4 Springer
}

\author{
Petros E. Carvounis
}

Received: 17 July 2014 / Accepted: 17 July 2014 / Published online: 31 July 2014

(C) Springer-Verlag Berlin Heidelberg 2014

Retinal and Choroidal Manifestations of Selected Systemic Diseases is a well-written and admirably well illustrated textbook. In the 27 book chapters, authors from across the globe who are leaders in the field cover the posterior segment manifestations of a wide range of systemic diseases including infectious diseases (e.g., toxoplasmosis, toxocariasis, viral diseases, cysticercosis, fungal and bacterial endophthalmitis etc.), autoimmune diseases (e.g., sarcoidosis, Adamantiades-Behçet disease, systemic lupus erythematosus, Vogt-Koyanagi-Harada syndrome), metabolic disorders (diabetes mellitus), drug toxicity due to systemic medications, genetic diseases (Marfan syndrome, phakomatoses), as well as neoplastic and paraneoplastic disease.

The chapters are well referenced and the information is up to date, clinically relevant, and presented in a clear manner. The goal is not to impart sterile knowledge but to allow the practicing clinician to provide better patient care. Clinical and imaging findings are admirably well illustrated. Each chapter has a penultimate section entitled "controversies and perspectives" that some of the authors have used well to address controversial diagnostic and management problems. A section entitled "focal points" concludes each chapter. Therein, key points are presented in bullet point or list form. Claims are generally supported by evidence in the peer-reviewed literature with instances of the authors omitting to recognize their biases few and far between. Recent developments in ancillary investigations including imaging and the use of serologic investigations including PCR are well covered. Treatment options are carefully considered and evidence to support them is put forth, but an authoritative direction is also helpfully provided.

In common with other multi-author textbooks, the depth of coverage of each topic, style of writing, ability to capture the reader's attention, and extent of evidence-based presentation is variable. However, there are no instances of poorly written chapters, topics superficially covered, or authoritative writing without adequate reference to supporting evidence. Therefore, while Retinal and Choroidal Manifestations of Selected Systemic Diseases could be used as a reference book, optimally, it should serve as a textbook to further and update the knowledge of keen ophthalmology trainees, practicing vitreoretinal specialists, uveitis specialists, or comprehensive ophthalmologists with an interest in posterior segment. The editor should be congratulated for producing a readable, highly informative, and clinically useful text.
P. E. Carvounis $(\bowtie)$

Cullen Eye Institute, Baylor College of Medicine, Alkek Eye Center, 1977 Butler Blvd, Houston, TX 77030, USA

e-mail: carvounis@yahoo.com 